\title{
Memristors Using Solution-Based IGZO Nanoparticles
}

\author{
Jose Rosa, ${ }^{\dagger, \|}$ Asal Kiazadeh, ${ }^{*}{ }^{\dagger}$ [i] Lídia Santos, ${ }^{\dagger, \perp}$ Jonas Deuermeier, $^{\dagger}{ }^{\circ}$ Rodrigo Martins, ${ }^{\dagger}$ \\ Henrique Leonel Gomes, ${ }^{\neq}, \S$ and Elvira Fortunato ${ }^{\dagger}$ (1) \\ ${ }^{\dagger}$ i3N/CENIMAT, Department of Materials Science, Faculty of Sciences and Technology, Universidade NOVA de Lisboa and \\ CEMOP/UNINOVA, Campus de Caparica, 2829-516 Caparica, Portugal \\ ${ }^{\ddagger}$ FCT, Universidade do Algarve, 8005-139 Faro, Portugal \\ ${ }^{\S}$ IT-Instituto de Telecomunicações, Av. Rovisco, Pais, 1, 1049-001 Lisboa, Portugal
}

Supporting Information

ABSTRACT: Solution-based indium-gallium-zinc oxide (IGZO) nanoparticles deposited by spin coating have been investigated as a resistive switching layer in metal-insulatormetal structures for nonvolatile memory applications. Optimized devices show a bipolar resistive switching behavior, low programming voltages of $\pm 1 \mathrm{~V}$, on/off ratios higher than 10, high endurance, and a retention time of up to $10^{4} \mathrm{~s}$. The better performing devices were achieved with annealing temperatures of $200{ }^{\circ} \mathrm{C}$ and using asymmetric electrode materials of titanium and silver. The physics behind the improved
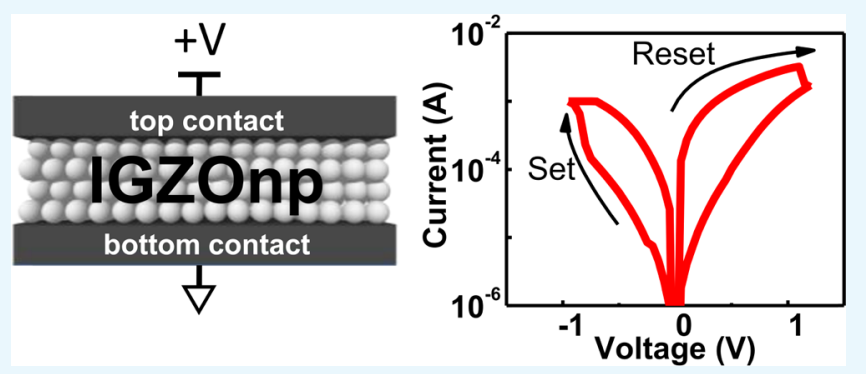
switching properties of the devices is discussed in terms of the oxygen deficiency of IGZO. Temperature analysis of the conductance states revealed a nonmetallic filamentary conduction. The presented devices are potential candidates for the integration of memory functionality into low-cost System-on-Panel technology.

\section{INTRODUCTION}

There are fundamental limits to the downscaling of materials involved in the current complementary metal-oxide-semiconductor technologies. Resistive random-access memories (RRAMs) can be considered as a highlight as they consist of two terminal devices with metal-insulator-metal (MIM) structure, allowing large-scale-integration circuits. ${ }^{1}$

They can be described, in simplified form, as a nonlinear passive resistor (memristor). RRAMs combine the best features of current memories such as the fast speed of SRAM, high density of DRAM, and nonvolatile properties of flash memory. These advantages support the idea that the future of memory technology will be based on RRAM devices. ${ }^{2-5}$ This memory technology will revolutionize all consumer electronic products from computing technology to healthcare and the Internet of Things.

An ideal System-on-Panel (SoP) technology utilizes electronic circuits, which are deposited with the same tools and materials as those used for the display components. This explains the choice to investigate amorphous indium-galliumzinc oxide (a-IGZO) for RRAMs, because it can be commercially applied in the thin-film transistors (TFTs) of driver circuits for active-matrix displays. ${ }^{6,7}$ The highly competitive markets for such displays, as mentioned above, demand the lowest production cost possible, which motivates a solution-based deposition route. Here, the main bottleneck is to find the optimal compromise between electrical performance/ reliability and production cost effectiveness.
Developments in IGZO-based RRAM have been reported elsewhere, focusing on studies of bipolar behavior in fully transparent devices, ${ }^{8,9}$ unipolar switching behavior, ${ }^{10}$ electrode influence, ${ }^{11}$ filament nature, ${ }^{12}$ and flexible devices. ${ }^{13}$ A single synaptic device based on an a-IGZO memristor has been reported, ${ }^{14}$ demonstrating the versatility of these devices. The most common industrially relevant deposition technique for IGZO films is radio frequency magnetron sputtering, and several ways to improve the performance of the corresponding memory devices have been reported. An interlayer of $\mathrm{Ti}$ at the interface improves the device yield and resistive switching properties due to the formation of $\mathrm{TiO}_{x}{ }^{15}$ Microwave irradiation can be used to obtain a smoother surface, which results in more stable resistive switching properties. ${ }^{16}$ By engineering the concentration of oxygen vacancy defects, stable forming-free resistive switching characteristics are obtained..$^{17}$ $\mathrm{SiO}_{2}$ has been used as a series resistance material to produce a thinner conductive filament. ${ }^{18}$ These reports show the excellent resistive memory properties that can be obtained with sputterdeposited IGZO. This justifies the investigation of low-cost fabrication routes such as deposition from solution, because such methods typically lead to more defective materials with poorer electrical properties. However, to the best of our knowledge, only two reports on solution-based IGZO nonvolatile memories exist so far: Inspired by low-cost IGZO

Received: August 11, 2017

Accepted: October 20, 2017

Published: November 29, 2017 


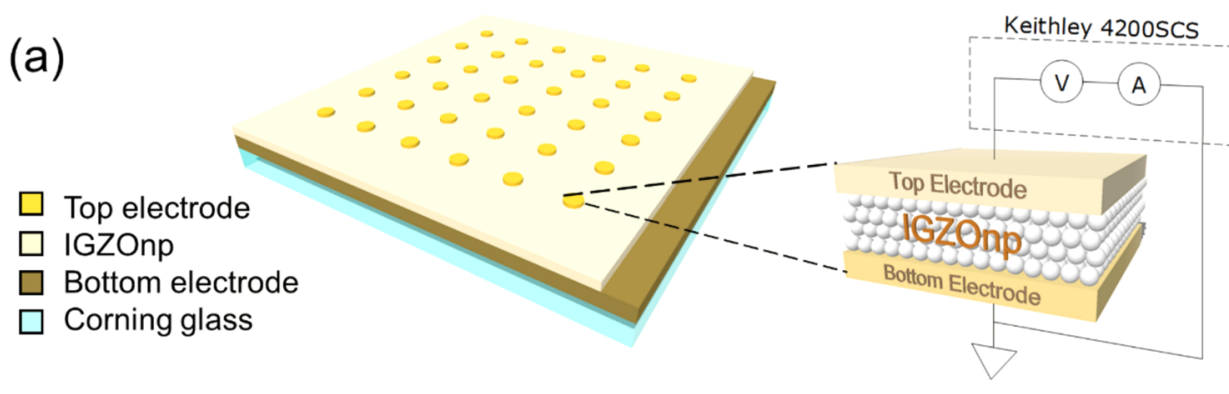

(c) IGZOnp/Ti $\left(350^{\circ} \mathrm{C}\right)$
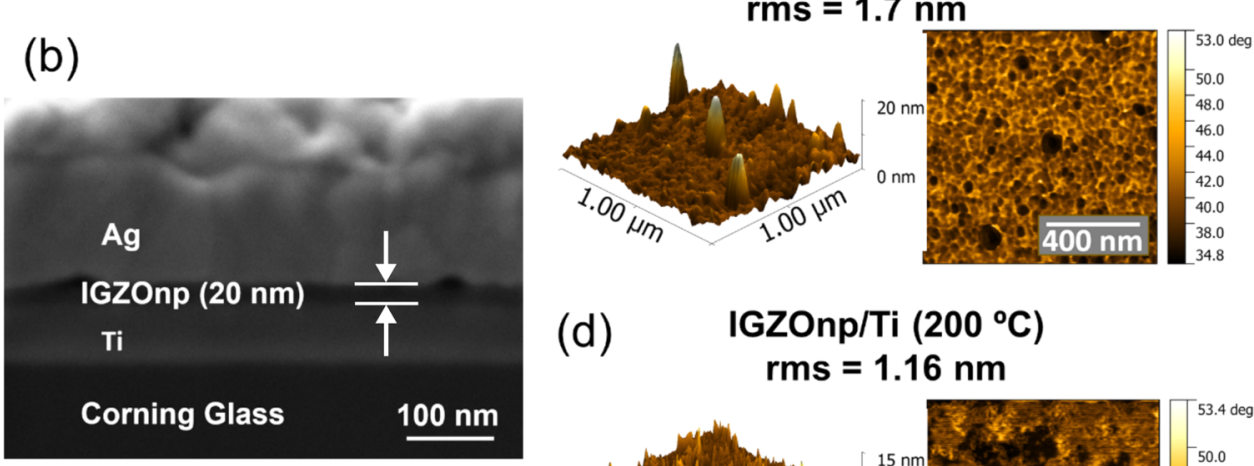

(d) IGZOnp/Ti $\left(200^{\circ} \mathrm{C}\right)$

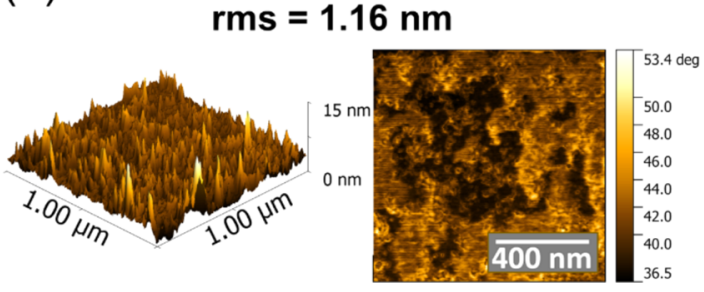

Figure 1. Device structure and IGZO morphology. (a) Typical $6 \times 6$ matrix fabricated on Corning glass and respective device schematic. (b) MIM structure cross section showing a $20 \mathrm{~nm}$ IGZOnp layer, arrows schematically indicate the IGZO thickness. Morphological characterization by AFM deflection images of $1 \times 1 \mu \mathrm{m}^{2}$ IGZOnp on Ti annealed at (c) $350{ }^{\circ} \mathrm{C}$ and (d) $200{ }^{\circ} \mathrm{C}$, on the left and respective phase images on the right.

solution-based TFTs, Kim et al. ${ }^{19}$ developed a-IGZO solutionbased bipolar RRAM. However, the devices operate at high current level and the reported on/off ratio did not exceed 1 order of magnitude. Later, $\mathrm{Hu}$ et $\mathrm{al}^{20}$ reported an a-IGZObased RRAM device fabricated by a photochemical solution deposition with improved unipolar memory performance.

This work intends to further explore the potential of solution-based synthesis of oxide-based RRAM. The here presented method comprises the synthesis of nanoparticles followed by thin-film deposition from a dispersion. An important reason to choose such a dual-stage process is the greater production flexibility compared to that of direct deposition from solution. To the best of our knowledge this is the first report on a RRAM device based on IGZO nanoparticles. Furthermore, a maximum process temperature as low as $200{ }^{\circ} \mathrm{C}$ is introduced because this enables the integration on flexible substrates.

\section{RESULTS AND DISCUSSION}

IGZO nanoparticles (IGZOnp) used as the memristive switching layer were produced by hydrothermal synthesis. The film was deposited on substrates using a common $\mathrm{Ti}$ bottom contact. The annealing temperature of $350{ }^{\circ} \mathrm{C}$ was optimized by Santos et al. $^{21}$ and applied to two substrates, the remaining two substrates were annealed at $200{ }^{\circ} \mathrm{C}$ for comparison.

For each annealing temperature, two types of MIM structures were fabricated. One structure is symmetric using
Ti top electrodes $(\mathrm{Ti} / \mathrm{IGZOnp} / \mathrm{Ti})$. The other structure is asymmetric by applying $\mathrm{Ag}$ as top contact ( $\mathrm{Ag} / \mathrm{IGZOnp} / \mathrm{Ti}$ ).

Figure 1 depicts a typical substrate with the 6 by 6 matrix of MIM structures used in this study. A cross-section view of the device by scanning electron microscopy (SEM) in Figure 1b shows the $20 \mathrm{~nm}$ thick IGZO nanoparticle layer. Furthermore, Figure 1c,d shows the surface morphology characterized by atomic force microscopy (AFM) of the IGZOnp thin-film deposited on $\mathrm{Ti}$ on Corning glass. A smaller nanoparticle size is observed for the lower temperature. For $200{ }^{\circ} \mathrm{C}$, the mean particle radius was approximately $3 \mathrm{~nm}$ whereas for $350{ }^{\circ} \mathrm{C}$, it was $\sim 4 \mathrm{~nm}$. It was also observed that for lower temperatures, there is a decrease of surface roughness.

As is often reported for resistive switching systems, the MIM structures undergo an irreversible electroforming process before they can act as a memory device. ${ }^{22}$ The original conduction state is referred to as the pristine state.

Figure 2 compares the current-voltage characteristic $(I-V)$ of a typical pristine state as well as off-state and on-state $I-V$ curves of all four devices. In the pristine state, samples annealed at $350{ }^{\circ} \mathrm{C}$ show higher conductance than that of the ones annealed at $200{ }^{\circ} \mathrm{C}$. When Ti is used for both electrodes, the I$V$ curve is symmetric for both polarities. In contrast, MIM structures using $\mathrm{Ag}$ as top contact electrode and $\mathrm{Ti}$ as bottom contact show a rectifying behavior in the pristine state.

To electroform the memory cell, particular care has to be taken to prevent the immediate breakdown, thus current compliance (CC) is required. For the Ti/IGZOnp/Ti devices, the forming step occurs by a positive voltage applied to the top 
(a) Ti/lGZOnp/Ti, $350^{\circ} \mathrm{C}$
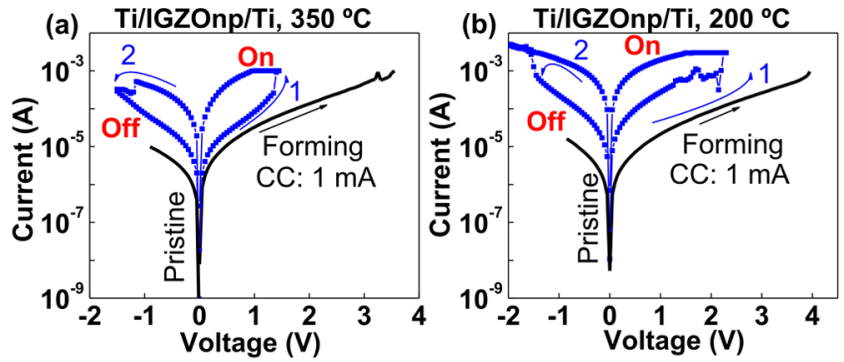

(c) Ag/lGZOnp/Ti, $350^{\circ} \mathrm{C}$
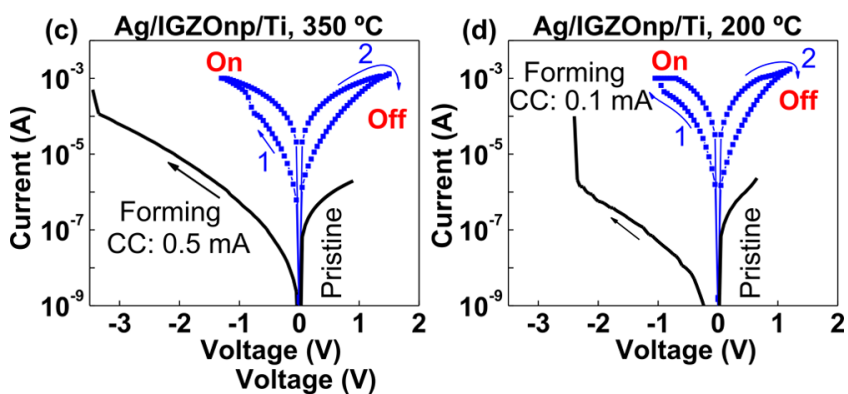

Figure 2. Typical $I-V$ characteristics showing the forming process and memory states. (a, b) Ti/IGZOnp/Ti annealed at 350 and $200{ }^{\circ} \mathrm{C}$, respectively. (c, d) Ag/IGZOnp/Ti annealed at 350 and $200{ }^{\circ} \mathrm{C}$, respectively.

electrode. However, it should be noted that structures with the same type of metal electrode $(\mathrm{Ti})$ at both contacts do not have a preferential voltage polarity to electroform, thus applying positive or negative voltages led to identical resistive switching behavior.

For the MIM structure using the silver top electrode, electroforming occurs only when a negative bias is applied to the silver contact. Forming under positive bias leads to unstable switching behavior after a few programming cycles. Furthermore, electroforming is established at lower voltages compared to that for the symmetric MIM structures using Ti on both top and bottom electrodes. Finally, the low conductance state (offstate) has a higher conductance than the pristine state.

Cycles of endurance and retention tests were carried out for all four devices to evaluate the impact of different electrodes and annealing temperature on memory performance. Reading voltages were $\pm 0.1 \mathrm{~V}$, corresponding to set polarization. Figure 3 shows endurance tests over 100 cycles in sweep voltage mode carried out for all four test devices. The Ti/IGZOnp/Ti device annealed at $350{ }^{\circ} \mathrm{C}$ shows a set voltage at $1.3 \mathrm{~V}$ in the first cycle, with a tendency to shift to higher voltages as the number of cycles increases. Noncontrollable intermediate switching events to off-state appear during the endurance cycles under the reset process between -0.8 and $-1.5 \mathrm{~V}$ (see Figure $3 \mathrm{a}$ ). For the $\mathrm{Ti} /$ IGZOnp/Ti device annealed at $200{ }^{\circ} \mathrm{C}$, set voltages between 0.8 and $1.3 \mathrm{~V}$ and reset voltages of -1 to $-1.5 \mathrm{~V}$ are observed (see Figure 3b). Here, the on/off current ratio and the memory performance gradually deteriorate after the $40^{\text {th }}$ cycle.

Figure 3c,d shows endurance cycle tests carried out on $\mathrm{Ag} /$ IGZOnp/Ti devices annealed at 350 and $200{ }^{\circ} \mathrm{C}$, respectively. The hysteresis shows a clockwise direction that is consistent with the negative bias forming loop. For the first device annealed at $350{ }^{\circ} \mathrm{C}$, the set process is performed at $-1.4 \mathrm{~V}$ and reset occurs at $2 \mathrm{~V}$. For the device annealed at $200{ }^{\circ} \mathrm{C}$, the set voltage is even lower, between -1 and $-0.8 \mathrm{~V}$, and the reset is obtained by applying a positive voltage of $1 \mathrm{~V}$. The operating on/off ratio window is more than 1 order of magnitude.

Concerning the data retention presented in Figure 3, the comparison of all four devices shows that all devices except $\mathrm{Ti}$ / IGZOnp/Ti fabricated at $200{ }^{\circ} \mathrm{C}$ annealing temperature have a retention time of at least $10^{4} \mathrm{~s}$ when keeping the switching margin equal to or even higher than 10 .

Table 1 compares the main electrical characteristics for both $\mathrm{Ti} /$ IGZOnp/Ti and Ag/IGZOnp/Ti memory devices annealed at different temperatures. The yield was defined here as the number of devices out of 36 that show at least 10 stable and consecutive endurance cycles.
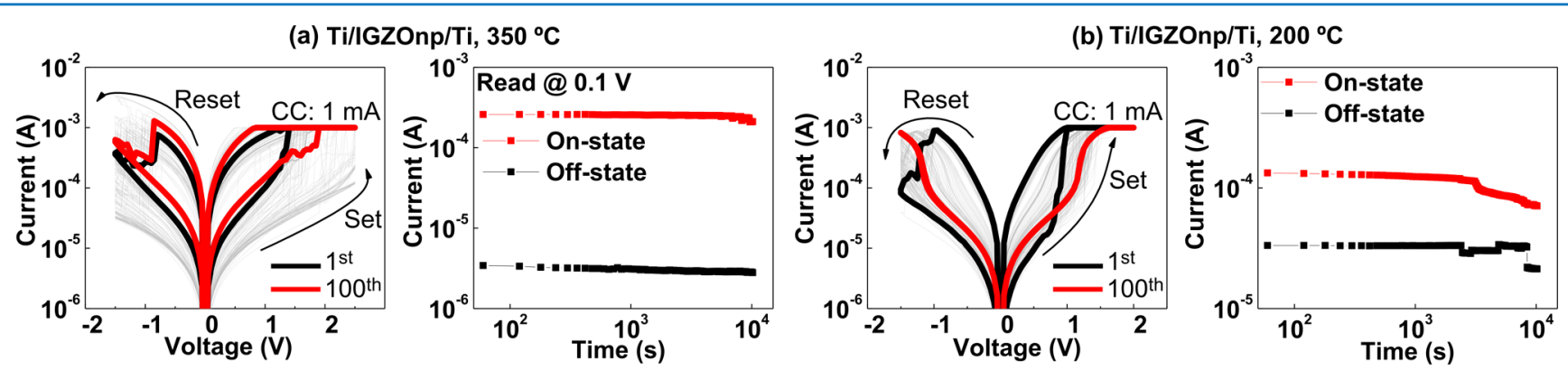

(c) Ag/IGZOnp/Ti, $350^{\circ} \mathrm{C}$
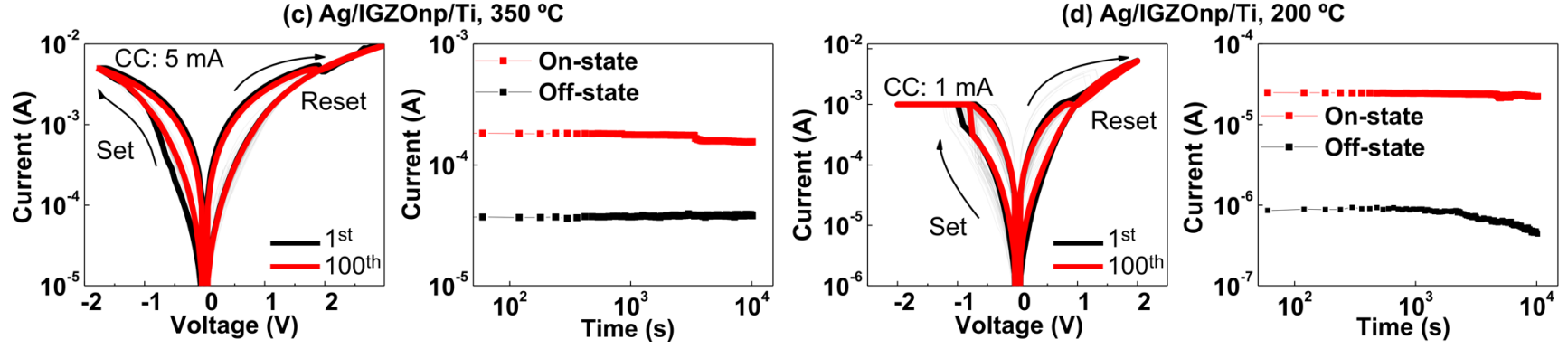

Figure 3. $I-V$ characteristic over 100 cycles and retention time characteristics at $\pm 0.1 \mathrm{~V}$ at room temperature for devices: (a) Ti/IGZOnp/Ti annealed at $350{ }^{\circ} \mathrm{C}$, (b) Ti/IGZOnp/Ti annealed at $200{ }^{\circ} \mathrm{C}$, (c) Ag/IGZOnp/Ti annealed at $350{ }^{\circ} \mathrm{C}$, and (d) Ag/IGZOnp/Ti annealed at $200{ }^{\circ} \mathrm{C}$. 
Table 1. Performance Comparison between Ti/IGZOnp/Ti and Ag/IGZOnp/Ti for 350 and $200{ }^{\circ} \mathrm{C}$ Annealing Temperatures

\begin{tabular}{|c|c|c|c|c|c|c|c|c|c|c|}
\hline structure & $T\left[{ }^{\circ} \mathrm{C}\right]$ & yield [\%] & $\mathrm{CC}^{a}[\mathrm{~mA}]$ & forming $[\mathrm{V}]$ & $\operatorname{set}^{b}[\mathrm{~V}]$ & $\operatorname{reset}^{b}[\mathrm{~V}]$ & read $[\mathrm{V}]$ & on/off ratio & retention $[\mathrm{s}]$ & endurance \\
\hline \multirow[t]{2}{*}{ Ti/IGZOnp/Ti } & 350 & 20 & 1 & +3.5 & +1.3 & -0.8 & +0.1 & $>10$ & $10^{4}$ & $80 / 100$ \\
\hline & 200 & 14 & 1 & +4.7 & +2.3 & -1.8 & +0.1 & $<10$ & $\sim 10^{4}$ & $40 / 100$ \\
\hline \multirow[t]{2}{*}{$\mathrm{Ag} / \mathrm{IGZOnp} / \mathrm{Ti}$} & 350 & 64 & 0.5 & -1.9 & -1.4 & +2 & -0.1 & 10 & $10^{4}$ & 100 \\
\hline & 200 & 68 & 0.01 & -1.5 & -1 & +1 & -0.1 & $>10$ & $>10^{4}$ & 100 \\
\hline
\end{tabular}

$\mathrm{Ag} / \mathrm{IGZOnp} / \mathrm{Ti}$ devices annealed at $200{ }^{\circ} \mathrm{C}$ provide the best memory performance. According to the data compared in Table 1, the Ag/IGZOnp/Ti structures show the highest yield $(68 \%)$, the higher data retention time, and operate at the lowest switching voltages. Furthermore, they are electroformed at very low current $(10 \mu \mathrm{A})$.

It is remarkable that the silver-based memory devices show a self-compliant behavior, i.e., set occurs prior to reaching the CC level. This can be observed when a rather high CC level is chosen, as shown in Figure 3c. Without the need for an external current compliance, the simpler circuit provides a unique advantage for high density memory cells in crossbar arrays. The origin of the self-compliant phenomenon remains elusive, even if some authors tentatively explain it as caused by the formation of a thin oxide layer between the switching layer and the contact. $^{18,23}$

For further understanding of the mechanism behind the reliable switching event of the $\mathrm{Ag} / \mathrm{IGZOnp} / \mathrm{Ti}$ device, the fitting of typical $I-V$ characteristics in a log-log scale was done (see Figure 4a). The space-charge-limited conduction is
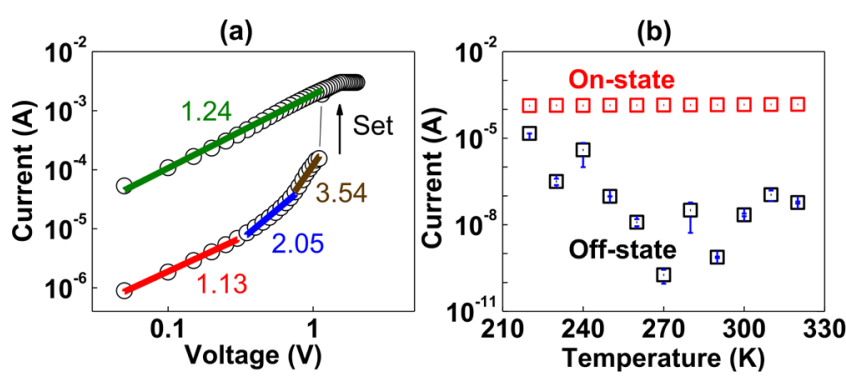

Figure 4. (a) $I-V$ characteristics of Ag/IGZOnp/Ti device plotted in $\log$-log scale, showing a space-charge-limited conduction mechanism, the numbers represent the slope. (b) Temperature dependence of the memory conductance states.

observed at the off-state, including three regions: (i) Ohmic region with an approximate slope close to 1 from 0 to $0.3 \mathrm{~V}$, (ii) a slope of 2 from 0.3 to $0.7 \mathrm{~V}$, which is in accordance with Child's square law $\left(I \propto V^{2}\right)$, and (iii) a steep increase region for voltages $>0.7 \mathrm{~V}$. The on-state shows Ohmic behavior, indicating the presence of highly conductive paths. The results are in agreement with the ones reported for a-IGZO memory devices. $^{9,20}$ Similar characteristics are observed for the $\mathrm{Ti}$ / IGZOnp/Ti structure, shown in Figure S2.

Figure $4 \mathrm{~b}$ shows the temperature dependence of the on- and off-states in the temperature range of $220-320 \mathrm{~K}$ at the read voltage of $-0.1 \mathrm{~V}$ for $\mathrm{Ag} / \mathrm{IGZOnp} / \mathrm{Ti}$ devices annealed at 200 ${ }^{\circ} \mathrm{C}$. The on-state conductance shows a weak thermal activation. This temperature dependence rules out metallic-like conduction and excludes that $\mathrm{Ag}$ atoms ${ }^{24}$ serve as the conducting filaments. Other reports of IGZO-based resistive memories have shown a metallic-like temperature dependence of conductance, $^{25-27}$ in contrast to the data presented here.
This may be related to residual impurities from the solution processing, more precisely ethylene glycol and ethanol. ${ }^{21}$ The presence of such impurities could be confirmed by attenuated total reflectance Fourier-transformed infrared spectroscopy (ATR-FTIR), see Figure S1. It has been shown that thermal activation of conductivity in IGZO with a high carrier concentration is related to the density of states in the vicinity of the valence band maximum. ${ }^{28,29}$ The nature of these states are $\mathrm{OH}$ groups, which act as compensating acceptor defects. $^{30,31}$ As observed by ATR-FTIR in Figure S1, the residual impurities from the solution processing introduce a considerable density of these defects in the material, which in turn hinder the Fermi energy to enter the conduction band and cause the observed nonmetallic temperature dependence of conductance.

The temperature dependence for the off-state shows an anomalous behavior. For the off-state below $270 \mathrm{~K}$, a very large positive temperature coefficient (PTC) is observed. The increase of the current upon lowering the temperature of the device is more than 2 orders of magnitude in a temperature range of $60 \mathrm{~K}\left(\alpha=0.02 \mathrm{~K}^{-1}\right)$. This PTC is anomalously large when compared with typical values for metals $\left(\alpha=4 \times 10^{-3}\right.$ $\mathrm{K}^{-1}$ for $\left.\mathrm{Ag}^{26}\right)$. This behavior has been observed on other RRAM devices and explained as due to filamentary conduction. ${ }^{32}$ For temperatures above $270 \mathrm{~K}$, the temperature dependence shows a semiconductor-like behavior. The transition point between the two behaviors occurs close to the temperature of the phase transition of water $(273 \mathrm{~K})$, which is unavoidably present due to the fabrication process. ${ }^{33}$

A stochastic electrical behavior is observed for the symmetric Ti-based device structure. By replacing the $\mathrm{Ti}$ top electrode with $\mathrm{Ag}$, the memristor shows stable switching properties with higher switching yield during endurance tests. In the following, the role of the titanium at the interface to the IGZO shall be discussed. Figure 5 serves as an accompanying graphical illustration. Titanium is referred to as an oxygen-getter material due to its high affinity for forming titanium oxide. ${ }^{34}$ In the devices presented here, this leads to the removal of oxygen from the IGZO layer. Because this process is accelerated at higher temperatures, the IGZOnp films annealed at $350{ }^{\circ} \mathrm{C}$ are more conductive than the ones annealed at $200{ }^{\circ} \mathrm{C}$. In Figure 5, this is represented by the more intense red color toward the titanium bottom electrode. Although the annealing is done in contact with ambient air, the electrical conductance of the devices is dominated by the oxygen removal at the titanium bottom electrode and not by an eventual oxidation at the IGZOnp film surface. The presence of an anatase phase could be confirmed by grazing incidence X-ray diffraction (XRD) in the samples annealed at $350{ }^{\circ} \mathrm{C}$, whereas the same could not be detected in the samples annealed at $200{ }^{\circ} \mathrm{C}$ (see Figure S3). This confirms the increased oxidation of the titanium bottom electrode when the samples are annealed at the higher temperature. 
(a)

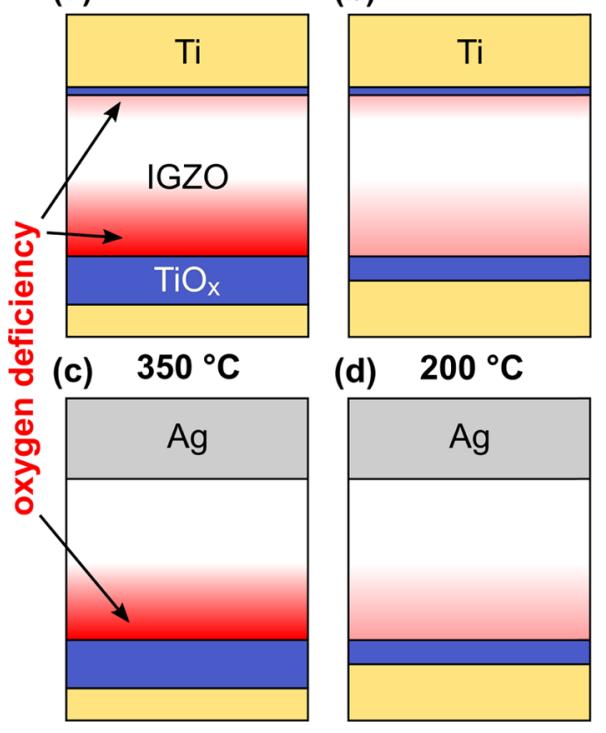

Figure 5. Schematic illustration of the pristine state of the different devices: $\mathrm{Ti} / \mathrm{IGZO} / \mathrm{Ti}$ annealed at (a) $350{ }^{\circ} \mathrm{C}$ and (b) $200^{\circ} \mathrm{C}$, and $\mathrm{Ag} /$ IGZO/Ti annealed at (c) $350{ }^{\circ} \mathrm{C}$ and (d) $200{ }^{\circ} \mathrm{C}$. The oxygen deficiency inside the IGZOnp layer is shown qualitatively in red color gradients.

The electron beam deposition of the titanium top electrode leads to the formation of a thin layer of oxidized titanium at the interface. ${ }^{35}$ Hence, a similar-although probably less severeoxygen-getter effect occurs at the top contact of the $\mathrm{Ti}$ / IGZOnp/Ti devices, which also leads to an increase of conductivity close to the top surface of the IGZO layer. In Figure 5, this is shown by the red color below the titanium top electrodes. When silver is used instead, the conductivity at the top interface is lower, because silver typically does not oxidize in contact with IGZO (based on the Gibbs free energies of formation $\left.^{34,36}\right)$. Moreover, silver is reported to form a Schottky barrier with IGZO, which implies a high resistivity in the IGZO at this interface. ${ }^{37,38}$ Therefore, under negative forming bias, a conductive filament based on oxygen vacancies growing from the bottom contact toward the top contact results in more stable switching events, as compared to the titanium top contact. Furthermore, the conductance in the pristine state under negative bias allows for the use of a lower CC during electroforming (see Table 1). In this way, the risk of breakdown due to Joule heating effects is minimized.

\section{CONCLUSIONS}

The solution-based $\mathrm{Ti} / \mathrm{IGZOnp} / \mathrm{Ti}$ and Ag/IGZOnp/Ti memory devices were fabricated at different annealing temperatures of 200 and $350{ }^{\circ} \mathrm{C}$. The lowest switching voltages were observed for $\mathrm{Ag} / \mathrm{IGZOnp} / \mathrm{Ti}$ devices annealed at $200{ }^{\circ} \mathrm{C}$ with set and reset voltages of +1 and $-1 \mathrm{~V}$, respectively, and retention times of up to $10^{4} \mathrm{~s}$. The Ag/IGZOnp/Ti devices showed higher yields compared to those of the symmetric Tibased devices. Temperature-dependent measurements reveal a nonmetallic conductive filament at the on-state.

It could be shown that the oxygen-getter effect due to the oxidation of titanium increases the conductivity of IGZO. If both IGZO interfaces are oxygen deficient, stochastic switching properties are observed. The solution is a nonsymmetric device architecture using a nonoxidizing top electrode such as silver. The resulting lower conductivity is favorable to establish a well- defined filamentary path by applying lower current compliance during electroforming.

In summary, we demonstrated an RRAM device processed from a dispersion of solution-based IGZO nanoparticles. In comparison to the previously reported works on solution-based IGZO ${ }^{20}$ we have achieved lower operation power. Also, selfcompliant operation is a major feature that simplifies the circuit design. This RRAM device has the potential to be integrated into SoP technology.

\section{EXPERIMENTAL SECTION}

4.1. Device Fabrication. IGZO nanoparticles were produced following a recipe previously developed by Santos et al. ${ }^{21}$ with gallium nitrate hydrate $\left(\mathrm{Ga}\left(\mathrm{NO}_{3}\right)_{3} \cdot \mathrm{H}_{2} \mathrm{O}\right.$, SigmaAldrich, 99.9\%), indium acetate $\left(\mathrm{In}\left(\mathrm{CH}_{3} \mathrm{COO}\right)_{3}\right.$, SigmaAldrich, 99.99\%), and zinc acetate $\left(\mathrm{Zn}\left(\mathrm{CH}_{3} \mathrm{COO}\right)_{3} \cdot 2 \mathrm{H}_{2} \mathrm{O}\right.$, Sigma-Aldrich, 99\%) precursors with a molar ratio of 3:6:2, respectively. The mixture was dissolved in 2-methoxyethanol (6 $\mathrm{mL}$, Sigma-Aldrich, 99,5\%) with ethanolamine (0.2 mL, Fluka, $98 \%)$ as a cation reductor, and the solution was left stirring at $50{ }^{\circ} \mathrm{C}$ for $1 \mathrm{~h}$. The final solution was transferred to the polytetrafluoroethylene cup of a $23 \mathrm{~mL}$ stainless-steel autoclave (4745 general purpose vessel, Parr), and placed in an oven (L3/11/B170, Nabertherm) at $180{ }^{\circ} \mathrm{C}$ for $24 \mathrm{~h}$. The resulting product was collected by centrifugation at $4000 \mathrm{rpm}$ for $5 \mathrm{~min}$ (F140, Focus instruments) and dispersed in ethanol $(20 \mathrm{~mL})$.

IGZOnp ink was ensured by mixing ethylene glycol (Carlo Erba, 95.5\%) with the previous dispersion in a 0.4:1.6 proportion, respectively, and consequently, the mixture was left stirring for $24 \mathrm{~h}$. Then, it was sonicated for $2 \mathrm{~min}$ and filtered with a $0.45 \mu \mathrm{m}$ porous diameter filter (Sartorius CA). The deposition process was performed by spin coating 4 layers at $2000 \mathrm{rpm}$ for $35 \mathrm{~s}$ and drying at $100{ }^{\circ} \mathrm{C}$ for 1 min between each layer. The annealing process was done in air after the fourth deposition at 350 and $200{ }^{\circ} \mathrm{C}$ for $1 \mathrm{~h}$.

MIM structures were fabricated on glass substrates (1737, Corning). First, a $60 \mathrm{~nm}$ Ti thin-film was deposited by e-beam evaporation (homemade apparatus) followed by spin coating deposition of the IGZO ink, creating an IGZOnp thin-film layer of approximately $20 \mathrm{~nm}$, see Figure $1 \mathrm{~b}$. The top electrode was deposited with the same procedure as the bottom electrode but using a shadow mask, where titanium $(60 \mathrm{~nm})$ or silver $(60$ $\mathrm{nm}$ ) were deposited on different substrates. A device area of $19.6 \mu \mathrm{m}^{2}$ was defined by top electrode deposition through a shadow mask.

4.2. Materials and Device Characterization. Structural and morphological characterization of the IGZO nanoparticles was performed by scanning electron microscopy (Carl Zeiss AURIGA). The nanoparticles were deposited on a silicon substrate for the X-ray diffraction (XRD) measurements and scanned in the $20-70^{\circ} 2 \theta$ range with a step size of $0.033^{\circ}$ in a PANalytical's X'Pert PRO MPD diffractometer with $\mathrm{Cu} \mathrm{K} \alpha$ radiation. In addition, the devices were measured by grazing incidence X-ray diffraction with the same diffractometer at an incident angle of $\Omega=1.1^{\circ}$ and a step size of $0.1^{\circ}$. The IGZO thin-film surface roughness was analyzed by atomic force microscopy in a commercial microscope, Asylum Research MFP-3D. Attenuated total reflectance Fourier-transformed infrared spectroscopy was measured with a Thermo Nicolet 6700 Spectrometer, using a single bounced diamond crystal $\left(4500-525 \mathrm{~cm}^{-1}\right)$.

IGZOnp memristor electrical characterization was performed using a semiconductor characterization system (Keithley 
4200SCS). Positive bias refers to the top electrode in the sandwich structure.

\section{ASSOCIATED CONTENT}

\section{S Supporting Information}

The Supporting Information is available free of charge on the ACS Publications website at DOI: 10.1021/acsomega.7b01167.

Confirmation of the presence of organic residues due to the solution process by attenuated total reflectance Fourier-transformed infrared spectroscopy; Spacecharge-limited conduction mechanism of the $\mathrm{Ti}$ / IGZOnp/Ti device; X-ray diffractograms of the synthesized IGZO nanoparticles and the $\mathrm{Ag} / \mathrm{IGZOnp} / \mathrm{Ti}$ devices after different annealing temperatures (PDF)

\section{AUTHOR INFORMATION}

\section{Corresponding Author}

*E-mail: a.kiazadeh@fct.unl.pt.

\section{ORCID}

Asal Kiazadeh: 0000-0002-8422-5762

Jonas Deuermeier: 0000-0002-2764-3124

Elvira Fortunato: 0000-0002-4202-7047

\section{Present Addresses}

${ }^{\perp}$ HOVIONE, Sete Casas, 2674-506 Loures, Portugal (L.S.).

"CeNTI, Rua Fernando Mesquita, 2785, 4760-034 VN Famalicão, Portugal (J.R.).

\section{Author Contributions}

The manuscript was written through contributions of all authors. All authors have given approval to the final version of the manuscript.

\section{Notes}

The authors declare no competing financial interest.

\section{ACKNOWLEDGMENTS}

This work is funded by National Funds through FCT Portuguese Foundation for Science and Technology, references UID/CTM/50025/2013 and SFRH/BDP/99136/2013 and FEDER funds through the COMPETE 2020 Programme under the project number POCI-01-0145-FEDER-007688.

\section{REFERENCES}

(1) Sakamoto, T.; Lister, K.; Banno, N.; Hasegawa, T.; Terabe, K.; Aono, M. Electronic Transport in $\mathrm{Ta}_{2} \mathrm{O}_{5}$ Resistive Switch. Appl. Phys. Lett. 2007, 91, No. 092110.

(2) Yang, J. J.; Strukov, D. B.; Stewart, D. R. Memristive Devices for Computing. Nat. Nanotechnol. 2013, 8, 13-24.

(3) Pan, F.; Gao, S.; Chen, C.; Song, C.; Zeng, F. Recent Progress in Resistive Random Access Memories: Materials, Switching Mechanisms, and Performance. Mater. Sci. Eng., R 2014, 83, 1-59.

(4) Choi, B. J.; Torrezan, A. C.; Strachan, J. P.; Kotula, P. G.; Lohn, A. J.; Marinella, M. J.; Li, Z.; Williams, R. S.; Yang, J. J. High-Speed and Low-Energy Nitride Memristors. Adv. Funct. Mater. 2016, 26, 52905296.

(5) Freitas, R.; Wilcke, W. Storage-Class Memory: The next Storage System Technology. IBM J. Res. Dev. 2008, 52, 439-447.

(6) Nomura, K.; Ohta, H.; Takagi, A.; Kamiya, T.; Hirano, M.; Hosono, H. Room-Temperature Fabrication of Transparent Flexible Thin-Film Transistors Using Amorphous Oxide Semiconductors. Nature 2004, 432, 488-492.

(7) Fortunato, E.; Barquinha, P.; Martins, R. Oxide Semiconductor Thin-Film Transistors: A Review of Recent Advances. Adv. Mater. 2012, 24, 2945-2986.
(8) Chen, M.-C.; Chang, T.-C.; Huang, S.-Y.; Chen, S.-C.; Hu, C.-W.; Tsai, C.-T.; Sze, S. M. Bipolar Resistive Switching Characteristics of Transparent Indium Gallium Zinc Oxide Resistive Random Access Memory. Electrochem. Solid-State Lett. 2010, 13, H191.

(9) Lo, C.-C.; Hsieh, T.-E. Forming-Free, Bipolar Resistivity Switching Characteristics of Fully Transparent Resistive Random Access Memory with IZO/ $\alpha$-IGZO/ITO Structure. J. Phys. D. Appl. Phys. 2016, 49, No. 385102.

(10) Kim, C. H.; Jang, Y. H.; Hwang, H. J.; Song, C. H.; Yang, Y. S.; Cho, J. H. Bistable Resistance Memory Switching Effect in Amorphous InGaZnO Thin Films. Appl. Phys. Lett. 2010, 97, No. 062109.

(11) Chen, M.-C.; Chang, T.-C.; Tsai, C.-T.; Huang, S.-Y.; Chen, S.C.; Hu, C.-W.; Sze, S. M.; Tsai, M.-J. Influence of Electrode Material on the Resistive Memory Switching Property of Indium Gallium Zinc Oxide Thin Films. Appl. Phys. Lett. 2010, 96, No. 262110.

(12) Kang, Y. H.; Lee, T. Il; Moon, K.-J.; Moon, J.; Hong, K.; Cho, J.H.; Lee, W.; Myoung, J.-M. Observation of Conductive Filaments in a Resistive Switching Nonvolatile Memory Device Based on Amorphous InGaZnO Thin Films. Mater. Chem. Phys. 2013, 138, 623-627.

(13) Wang, Z. Q.; Xu, H. Y.; Li, X. H.; Zhang, X. T.; Liu, Y. X.; Liu, Y. C. Flexible Resistive Switching Memory Device Based on Amorphous InGaZnO Film With Excellent Mechanical Endurance. IEEE Electron Device Lett. 2011, 32, 1442-1444.

(14) Wang, Z. Q.; Xu, H. Y.; Li, X. H.; Yu, H.; Liu, Y. C.; Zhu, X. J. Synaptic Learning and Memory Functions Achieved Using Oxygen Ion Migration/Diffusion in an Amorphous InGaZnO Memristor. Adv. Funct. Mater. 2012, 22, 2759-2765.

(15) Fan, Y. S.; Liu, P. T.; Hsu, C. H. Investigation on Amorphous InGaZnO Based Resistive Switching Memory with Low-Power, HighSpeed, High Reliability. Thin Solid Films 2013, 549, 54-58.

(16) Hwang, Y.; An, H.; Cho, W. Performance Improvement of the Resistive Memory Properties of InGaZnO Thin Films by Using Microwave Irradiation. Jpn. J. Appl. Phys. 2014, 53, No. 04EJ04.

(17) Pei, Y.; Mai, B.; Zhang, X.; Hu, R.; Li, Y.; Chen, Z.; Fan, B.; Liang, J.; Wang, G. Forming Free Bipolar ReRAM of Ag/a-IGZO/Pt with Improved Resistive Switching Uniformity Through Controlling Oxygen Partial Pressure. J. Electron. Mater. 2015, 44, 645-650.

(18) Pei, Y.; Mai, B.; Zhang, X.; Hu, R.; Li, Y.; Chen, Z.; Fan, B.; Liang, J.; Wang, G. Performance Improvement of Amorphous IndiumGallium-Zinc Oxide ReRAM with $\mathrm{SiO}_{2}$ Inserting Layer. Curr. Appl. Phys. 2015, 15, 441-445.

(19) Kim, M. S.; Hwan Hwang, Y.; Kim, S.; Guo, Z.; Moon, D.-Il; Choi, J. M.; Seol, M. L.; Bae, B. S.; Choi, Y. K. Effects of the Oxygen Vacancy Concentration in InGaZnO-Based Resistance Random Access Memory. Appl. Phys. Lett. 2012, 101, No. 243503.

(20) Hu, W.; Zou, L.; Chen, X.; Qin, N.; Li, S.; Bao, D. Highly Uniform Resistive Switching Properties of Amorphous InGaZnO Thin Films Prepared by a Low Temperature Photochemical Solution Deposition Method. ACS Appl. Mater. Interfaces 2014, 6, 5012-5017.

(21) Santos, L.; Nunes, D.; Calmeiro, T.; Branquinho, R.; Salgueiro, D.; Barquinha, P.; Pereira, L.; Martins, R.; Fortunato, E. Solvothermal Synthesis of Gallium-Indium-Zinc-Oxide Nanoparticles for Electrolyte-Gated Transistors. ACS Appl. Mater. Interfaces 2015, 7, 638-646.

(22) Joshua Yang, J.; Miao, F.; Pickett, M. D.; Ohlberg, D. A. A.; Stewart, D. R.; Lau, C. N.; Williams, R. S. The Mechanism of Electroforming of Metal Oxide Memristive Switches. Nanotechnology 2009, 20, No. 215201.

(23) Maikap, S.; Jana, D.; Dutta, M.; Prakash, A. Self-Compliance RRAM Characteristics Using a Novel $\mathrm{W} / \mathrm{TaO}_{\mathrm{x}} / \mathrm{TiN}$ Structure. Nanoscale Res. Lett. 2014, 9, 292.

(24) Bid, A.; Bora, A.; Raychaudhuri, A. K. Temperature Dependence of the Resistance of Metallic Nanowires (Diameter $\geq 15 \mathrm{~nm}$ ): Applicability of Bloch-Grüneisen Theorem. Phys. Rev. B 2006, 74, 1-8.

(25) Wang, Z. Q.; Xu, H. Y.; Li, X. H.; Zhang, X. T.; Liu, Y. X.; Liu, Y. C. Flexible Resistive Switching Memory Device Based on Amorphous InGaZnO Film with Excellent Mechanical Endurance. IEEE Electron Device Lett. 2011, 32, 1442-1444.

(26) Wang, Z. Q.; Xu, H. Y.; Zhang, L.; Li, X. H.; Ma, J. G.; Zhang, X. T.; Liu, Y. C. Performance Improvement of Resistive Switching 
Memory Achieved by Enhancing Local-Electric-Field near Electromigrated Ag-Nanoclusters. Nanoscale 2013, 5, 4490-4494.

(27) Liu, S.; Zhao, X.; Li, Q.; Li, N.; Wang, W.; Liu, Q.; Xu, H. Analysis of the Negative-SET Behaviors in $\mathrm{Cu} / \mathrm{ZrO}_{2} / \mathrm{Pt}$ Devices. Nanoscale Res. Lett. 2016, 11, 542.

(28) Nomura, K.; Kamiya, T.; Yanagi, H.; Ikenaga, E.; Yang, K.; Kobayashi, K.; Hirano, M.; Hosono, H. Subgap States in Transparent Amorphous Oxide Semiconductor, In- $\mathrm{Ga}-\mathrm{Zn}-\mathrm{O}$, Observed by Bulk Sensitive X-Ray Photoelectron Spectroscopy. Appl. Phys. Lett. 2008, 92, No. 202117.

(29) Kamiya, T.; Nomura, K.; Hosono, H. Electronic Structures Above Mobility Edges in Crystalline and Amorphous In-Ga-Zn-O: Percolation Conduction Examined by Analytical Model. J. Disp. Technol. 2009, 5, 462-467.

(30) Robertson, J.; Guo, Y. Light Induced Instability Mechanism in Amorphous InGaZn Oxide Semiconductors. Appl. Phys. Lett. 2014, 104, No. 162102.

(31) Ide, K.; Kishida, Y.; Ueda, S.; Ohashi, N.; Hiramatsu, H.; Hosono, H.; Kamiya, T. P-13: Quantitative Analysis and Deconvolution of Subgap States in Amorphous In-Ga-Zn-O. SID Int. Symp. Dig. Tech. Pap. 2017, 48, 1273-1275.

(32) Gomes, H. L.; Rocha, P. R. F.; Kiazadeh, A.; De Leeuw, D. M.; Meskers, S. C. J. Anomalous Temperature Dependence of the Current in a Metal-Oxide-Polymer Resistive Switching Diode. J. Phys. D. Appl. Phys. 2011, 44, No. 025103.

(33) Han, S.; Choi, M. Y.; Kumar, P.; Stanley, H. E. Phase Transitions in Confined Water Nanofilms. Nat. Phys. 2010, 6, 685689.

(34) Ji, H.; Hwang, A. Y.; Lee, C. K.; Yun, P. S.; Bae, J. U.; Park, K. S.; Jeong, J. K. Improvement in Field-Effect Mobility of Indium Zinc Oxide Transistor by Titanium Metal Reaction Method. IEEE Trans. Electron Devices 2015, 62, 1195-1199.

(35) Kwang-Hyuk, C.; Han-Ki, K. Correlation between Ti source/ drain contact and performance of InGaZnO-based thin film transistors. Appl. Phys. Lett. 2013, 102, No. 052103.

(36) Dean, J. A. Lange's Handbook of Chemistry, 15th ed.; McGrawHill: New York, 1999.

(37) Trinh, T. T.; Nguyen, V. D.; Nguyen, H. H.; Raja, J.; Jang, J.; Jang, K.; Baek, K.; Dao, V. A.; Yi, J. Operation Mechanism of Schottky Barrier Nonvolatile Memory with High Conductivity InGaZnO Active Layer. Appl. Phys. Lett. 2012, 100, No. 143502.

(38) No, Y.; Yang, J.; Park, D.; Kim, T.; Choi, J.-W.; Choi, W. Improved Electrical Properties of Indium Gallium Zinc Oxide ThinFilm Transistors by AZO/Ag/AZO Multilayer Electrode. J. Sens. Sci. Technol. 2013, 22, 105-110. 